\section{Gold nanorods for fluorescence lifetime imaging in biology}

\author{
Yinan Zhang, ${ }^{a}$ Jun $\mathrm{Yu}^{\mathrm{b}}{ }^{\mathrm{b}}$ David J. S. Birch, ${ }^{\mathrm{a}}$ and \\ Yu Chen ${ }^{\mathrm{a}, *}$ \\ a University of Strathclyde, SUPA - Scottish Universities \\ Physics Alliance, Department of Physics, Centre \\ for Molecular Nanometrology, Photophysics Group, \\ John Anderson Building, 107 Rottenrow, Glasgow, G4 0NG \\ United Kingdom \\ ${ }^{b}$ University of Strathclyde, Royal College, Strathclyde \\ Institute of Pharmacy and Biomedical Sciences, 204 George \\ Street, Glasgow, G1 1XW United Kingdom
}

\begin{abstract}
Two-photon luminescence (TPL) from gold nanorods shows considerable potential in biological imaging. We study the imaging of gold nanorods in MadinDarby canine kidney (MDCK) cells using fluorescence lifetime imaging microscopy (FLIM). FLIM provides images with better contrast and sensitivity than intensity imaging. The characteristic fluorescence lifetime of gold nanorods is found to be less than 100 ps, which can be used to distinguish gold nanorods from other fluorescent labels and endogenous fluorophores in lifetime imaging. () 2010 Society of Photo-Optical Instrumentation Engineers. [DOI: 10.1117/1.3366646]
\end{abstract}

Keywords: gold nanorods; two-photon luminescence; fluorescence lifetime imaging microscopy.

Paper 10015LR received Jan. 14, 2010; revised manuscript received Feb. 20, 2010; accepted for publication Feb. 23, 2010; published online Apr. 2, 2010.

Gold nanorods are of great interest for optical imaging due to their remarkable absorption and scattering in the visible and near-infrared (NIR) regions enhanced by surface plasmon resonance (SPR). ${ }^{1,2}$ NIR band absorption between 700 and $900 \mathrm{~nm}$, a spectral window that permits photons to penetrate biological tissues with relatively high transmission, induces two-photon luminescence with strong intensity., ${ }^{3,4}$ Two-photon luminescence (TPL) from gold nanorods has been found to be sensitive to the polarization of the incident excitation. ${ }^{4,5}$ All these properties make gold nanorods attractive probes for invitro and in-vivo imaging., ${ }^{4,6,7}$ But so far, most related work has utilized traditional microscopy methods such as confocal microscopy and near-field optical microscopy imaging. ${ }^{8-12}$

In contrast to traditional imaging methods based on fluorescence intensity, fluorescence lifetime imaging microscopy (FLIM) provides contrast according to the fluorescence decay time, with the term "fluorescence" usually being associated with aromatic dye molecules. Here we have an inorganic system for which the term "luminescence" is usually applied. Lifetime imaging can be integrated with confocal microscopy, two-photon excitation microscopy, and other microscope systems. The luminescence (or fluorescence) decay time is the average time a fluorophore remains in the excited state after excitation. It does not change on intensity variations, and

*Address all correspondence to: Yu Chen. Tel: 44-141-5483087; Fax: 44-1415522891; E-mail: y.chen@strath.ac.u therefore lifetime measurements are not dependent on the local concentration of fluorophores, bleaching, the optical path of the microscope, the local excitation light intensity, or on the local luminescence detection efficiency. Also, the fluorescence decay time for aromatic molecules usually depends usefully on the intrinsic characteristics of the fluorophore and local environment (the local viscosity, $\mathrm{pH}$, or refractive index ${ }^{13-15}$ ) as well as interactions with other molecules, such as collisional or energy transfer quenching. ${ }^{16,17}$ Thus, as well as being able to distinguish spectrally overlapping fluorophores, ${ }^{18}$ imaging of the fluorescence lifetime can be used to probe the surroundings and dynamical processes of a fluorophore. ${ }^{19,20}$ Unlike electron microscopy, fluorescence/ luminescence techniques can be used in situ.

In this work, we use FLIM to visualize gold nanorods taken up by Madin-Darby canine kidney (MDCK) cells. A very short luminescence decay time of TPL from gold nanorods is observed. Compared with the lifetime of 4'-6-diamidino-2-phenylindole (DAPI), which is more than 2 ns, lifetime imaging shows good contrast. Furthermore, besides emission, the lifetime could be an alternative identification of gold nanorods in biological imaging.

Gold nanorods were synthesized by the seeded growth method. $^{21} 2.5-\mathrm{ml} \mathrm{HAuCl}_{4} \times 3 \mathrm{H}_{2} \mathrm{O}(0.001 \mathrm{M})$ and $0.6-\mathrm{ml}$ ice-cold $\mathrm{NaBH}_{4}(0.01 \mathrm{M})$ were added into $7.5-\mathrm{ml}$ cetyltrimethylammonium bromide (CTAB) $(0.1 \mathrm{M})$ to prepare the seed solution. The growth solution was synthesized by adding 0.15-M benzyldimethylammoniumchloride (BDAC), 50-ml $\mathrm{HAuCl}_{4} \times 3 \mathrm{H}_{2} \mathrm{O}(0.001 \mathrm{M}), 2-\mathrm{ml} \mathrm{AgNO}_{3}(0.004 \mathrm{M})$, and $700-\mu \mathrm{l}$ ascorbic acid $(0.778 \mathrm{M})$ to $50-\mathrm{ml} \mathrm{CTAB}$ solution $(0.1 \mathrm{M})$. Then $80-\mu \mathrm{l}$ seed solution ( $2 \mathrm{~h}$ after preparation) was injected into growth solution to grow gold nanorods. The absorption spectrum shows a longitudinal plasmon mode centered at around $750 \mathrm{~nm}$, and a weak transverse plasmon mode at $550 \mathrm{~nm}$.

Gold nanorod dispersions were centrifuged to remove the excess $\mathrm{CTAB}$ and redispersed in deionized water twice $(14,000 \mathrm{rpm}, 5 \mathrm{~min}$ per cycle) with a final optical density about 1.0 at $750 \mathrm{~nm}$. MDCK cells were treated with $100 \mu \mathrm{l}$ of gold nanorod solution and incubated for $3 \mathrm{~h}$ under standard cell culture conditions at $37{ }^{\circ} \mathrm{C}$ and $5 \% \mathrm{CO}_{2}$. The cells were washed thoroughly with phosphate buffered saline (PBS) to remove excess nanorods and fixed with $3.7 \%$ paraformaldehyde. After staining with DAPI, the sample was dispersed on a glass slide and covered with a coverslip for imaging.

FLIM was performed by using a confocal microscope (LSM 510, Carl Zeiss) equipped with a time-correlated single-photon counting (TCSPC) module (SPC-830, Becker and Hickl GmbH, Berlin, Germany). A femtosecond Ti:sapphire laser (Chameleon, Coherent, Santa Clara, California) was tuned at $750 \mathrm{~nm}$ to generate TPL from gold nanorods as well as DAPI. The laser pulse has a repetition rate of $80 \mathrm{MHz}$ and a duration of less than $200 \mathrm{fs}$. Emission was collected by a $60 \times$ water-immersion objective $(\mathrm{NA}=1.0)$ and a bandpass filter with a transmission window from 535 to $590 \mathrm{~nm}$.

Gold nanorods were examined prior to the cell uptake experiment. The pseudocolor TPL image of separated gold nanorods on a glass slide is shown in Fig. 1(a), with brighter spots considered to be clusters of nanorods. Furthermore,

1083-3668/2010/15(2)/020504/3/\$25.00 @ 2010 SPIE 
(a)

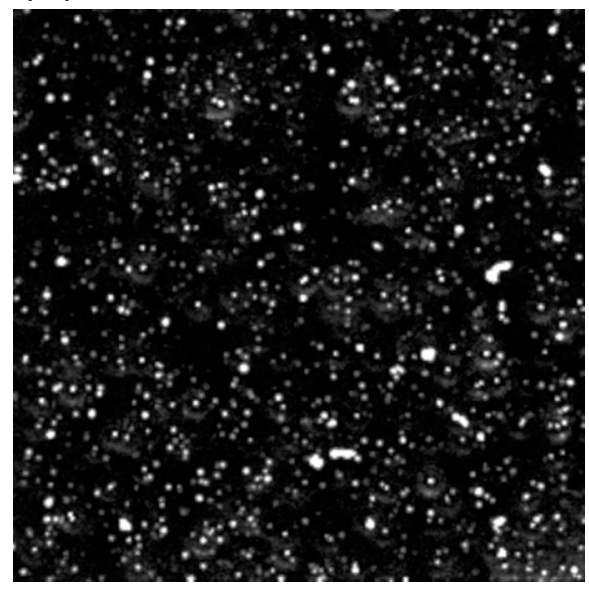

(b)

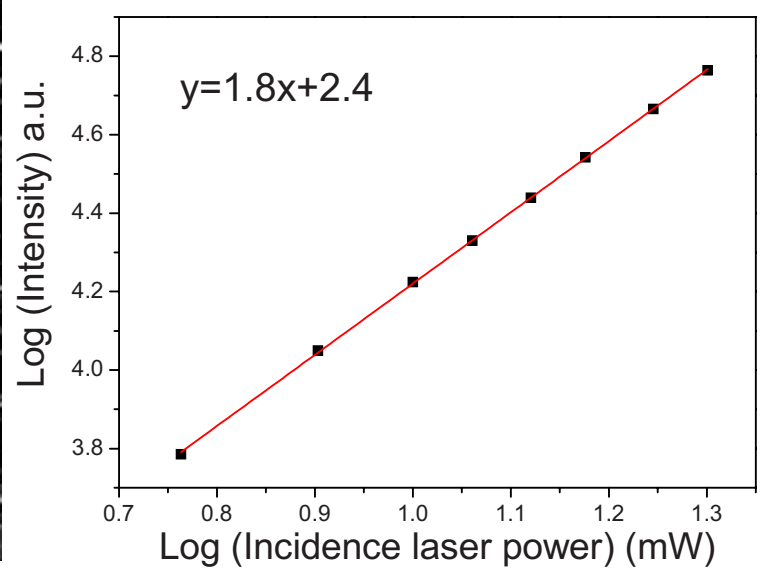

Fig. 1 (a) TPL image of gold nanorods dispersed on a glass slide, with a scanning area $133 \times 133 \mu \mathrm{m}$. (b) Dependence of luminescence intensity on incidence laser power (in log scale).

strong luminescence and a quadratic dependence on the incidence power were observed in a spectroscopy study of gold nanorods in bulk solution. The nonlinear property of the luminescence was studied by measuring the luminescence intensity as a function of excitation power. Luminescence was collected while increasing incident pulse power from 6 to $20 \mathrm{~mW}$. Figure 1(b) shows a typical result. A quadratic dependence of the signal intensity on the input power confirmed the luminescence originating from a two-photon excitation process.

Figure 2 compares the intensity and lifetime imaging of gold nanorods in cells. The elliptical shapes in Figs. 2(a) and 2(b) correspond to nuclei stained by DAPI (emission maximizes at around $460 \mathrm{~nm}$ and does not shift much under two photon excitation ${ }^{22}$ ), and bright spots are due to gold nanorods. Internalization of CTAB stabilized gold nanorods in cells has been reported before and studied using 3-D stack confocal microscopy. ${ }^{8,9}$ Here, compared with the cell reference sample [Fig. 2(a)], the luminescence intensity image shows that most nanorods were around DAPI dyed nuclei of MDCK cells, and that the nanorods have a stable and strong luminescence intensity. Figure 2(c) is a FLIM image from the same sample area as in Fig. 2(b), with different coded colors representing different lifetime scales. The luminescence lifetime of each pixel is obtained by applying a single or multiexponential fit; in this case a single exponential model was used. The fluorescence lifetime of DAPI is found to be longer than 2 ns and fitted well by a single exponential model. This value is in accordance with published data on FLIM analysis of DAPI. ${ }^{12}$ The TPL decay time of gold nanorods is shorter than 100 ps; this result is confirmed by FLIM study of pure gold nanorods on a glass slide, as well as time-resolved luminescence measurement of gold nanorods in a cuvette using the same excitation and time-correlated single-photon counting (TCSPC) $^{23}$ (not shown here). This value is much longer than those achieved in pump-probe and up-conversion research, where the lifetime of excited electrons in gold nanorods was found to be less than several picoseconds. ${ }^{9,24,25}$ This is probably due to the limit of time resolution caused by the instrumental response function of the TCSPC system, which is also around 100 ps. Our measurements thus give the upper limit of the decay time of the TPL process. The lifetime of TPL from large gold nanorods was reported to be 0.8 to $2.0 \mathrm{~ns}$, depending on the shape and size of the particles and also on the detection wavelength, but the details are not clear. ${ }^{5}$ Therefore, further research is required to reveal the full information of the dynamics of the TPL process in gold nanorods. Neverthe- (a)

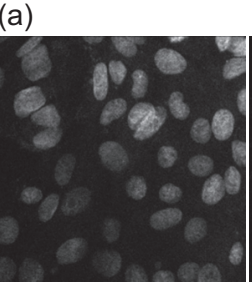

(d)

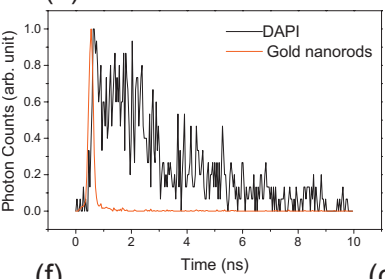

(f)

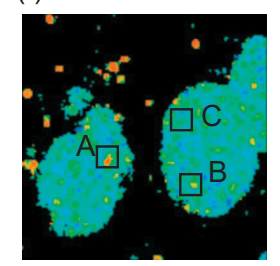

(b)

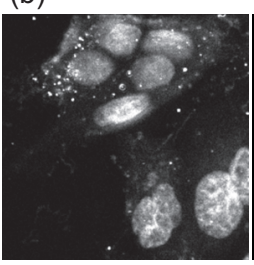

(c)

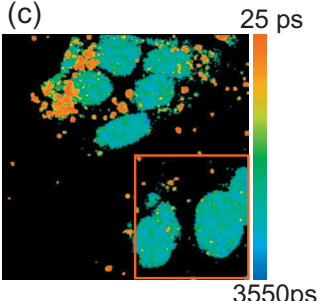

(e)

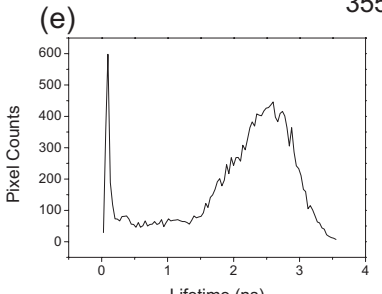

(g)

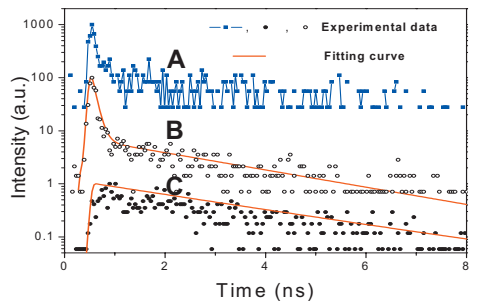

Fig. 2 (a) Intensity image of reference MDCK cells; (b) TPL intensity and (c) lifetime image of gold nanorods in MDCK cells; (d) typical TPL decay curves of DAPI and gold nanorods; (e) histogram of lifetime of every single pixel in the FLIM image; (f) enlarged lifetime image corresponding to the red rectangular area in (c); and (g) decay curves and fittings derived from regions $A, B$, and $C$ in ( $\mathrm{f}$ ). Scanning area: $133 \times 133 \mu \mathrm{m}$ for (a), and $67 \times 67 \mu \mathrm{m}$ for (b). 
less, the contrast in lifetime scale can be clearly seen in Fig. 2(c), which gives the typical line shapes of fluorescence decay curves of DAPI (black) and gold nanorods (red), as shown in Fig. 2(d). The luminescence lifetime distribution of all the pixels is shown in Fig. 2(e), where two peaks can be identified, one at around $100 \mathrm{ps}$ corresponding to gold nanorods, and the other to DAPI.

The FLIM image is in good accord with the intensity image, but is superior in contrast to the latter, which is caused by the huge gap between the decay time from the gold nanorods and DAPI. As the lifetime of gold nanorods is much shorter than that of commonly used fluorescent molecules and other quantum particles, the lifetime can be a useful identification parameter in bioimaging. Moreover, FLIM provides details that are lost when using traditional imaging due to the low luminescence intensity, because lifetime measurement does not depend on the fluorophore concentration. For example, detailed features from gold nanorods in the rectangular region in the FLIM image, Fig. 2(c), are apparent but not visible in the intensity image, Fig. 2(b). Further investigation on the dynamics of the TPL of gold nanorods and the influence of size and shape of the nanoparticles on the luminescence decay processes are underway.

FLIM can also provide more information based on luminescence lifetime analysis. Figure 2(f) amplifies the red rectangular region in Fig. 2(c). In the nuclei areas, orange dots and yellow areas are clearly visible surrounded by blue areas (DAPI). Three typical regions are labeled as A, B, and C. Corresponding decay curves of these three regions derived from the FLIM image are shown in Fig. 2(g), together with monoexponential (C) or biexponential (B) fittings. Typically, DAPI has a long lifetime in the range of 1.5 to $3.5 \mathrm{~ns}$, such as that found in region $\mathrm{C}$. In contrast, the decay curve in region $\mathrm{A}$ is in the system response level, similar to that from separated gold nanorods, indicating a lifetime shorter than 100 ps. The decay curve from region $\mathrm{B}$, which can be well fitted by a biexponential model with one component around 100 ps and the other at $3 \mathrm{~ns}$, are considered to be an overlay of the emissions of nanorods and DAPI. Thus the FLIM image reveals the existence of gold nanorods in these orange-yellow areas, indicating the uptake of gold nanorods by MDCK cells.

In summary, we have studied gold nanorods as luminescence labels in MDCK cells by FLIM, which provides a better contrast ratio and more detailed features than with intensity imaging. The luminescence lifetime of gold nanorods is found to be less than $100 \mathrm{ps}$, much shorter than most dye molecules, suggesting the possibility of distinguishing gold nanorods from extrinsic and endogenous fluorophores in biological systems. Thus, the characteristic lifetime, together with the polarization of TPL from gold nanorods, can be a promising imaging contrast agent for use in luminescence microscopy in biology.

\section{Acknowledgments}

The authors acknowledge J. Sutter for assistance during the experimental work. This work was supported by an EPSRC Science and Innovation Award, and a Pump Priming Award from SPIBS University of Strathclyde.

\section{References}

1. S. Link and M. El-Sayed, Int. Rev. Phys. Chem. 19(3), 409-453 (2000).

2. M. B. Mohamed, V. Volkov, S. Link, and M. A. El-Sayed, Chem. Phys. Lett. 317, 517-523 (2000).

3. R. A. Farrer, F. L. Butterfield, V. W. Chen, and J. T. Fourkas, Nano Lett. 5(6), 1139-1142 (2005).

4. H. Wang, T. B. Huff, D. A. Zweifel, W. He, P. S. Low, A. Wei, and J. Cheng, Proc. Natl. Acad. Sci. U.S.A. 102(44), 15752-15756 (2005).

5. K. Imura, T. Nagahara, and H. M. Okamoto, J. Phys. Chem. B 109(27),13214-13220 (2005).

6. X. H. Huang, I. H. El-Sayed, W. Qian, and M. A. El-Sayed, J. Am. Chem. Soc. 128(6), 2115-2120 (2006).

7. H. Ding, K. T. Yong, I. Roy, H. E. Pudavar, W. C. Law, E. J. Bergey, and P. N. Prasad, J. Phys. Chem. C 111(34), 12552-12557 (2007).

8. T. B. Huff, M. N. Hansen, Y. Zhao, J. Cheng, and A. Wei, Langmuir 23, 1596-1599 (2007)

9. T. B. Huff, M. N. Hansen, Y. Zhao, J. Cheng, and A. Wei, Nanomed. 2, 125-132 (2007)

10. N. J. Durr, T. Larson, D. K. Smith, B. A. Korgel, K. Sokolov, and A. Ben-Yakar, Nano Lett. 7(4), 941-945 (2007).

11. K. Imura, T. Nagahara, and H. M. Okamoto, J. Phys. Chem. B 108, 16344-16347 (2004).

12. L. Tong, Q. Wei, A. Wei, and J. Cheng, Photochem. Photobiol. 85, 21-32 (2009).

13. S. T. Hess, E. D. Sheets, A. Wagenknecht-Wiesner, and A. A. Heikal, Biophys. J. 85, 2566-2580 (2003).

14. B. Wilhelmi, Chem. Phys. 66, 351-355 (1982).

15. K. Suhling, P. M. W. French, and D. Phillips, Photobio. Sci. 4, 13-22 (2005).

16. J. R. Lakowicz, Principles of Fluorescence Spectroscopy, 2nd ed., Kluwer Academic-Plenum Publishers, New York, (1999).

17. B. Valeur, Molecular Fluorescence, Wiley-VCH, New York (2002).

18. A. Rück, C. H. Hülshoff, I. Kinzler, W. Becker, and R. Steiner, Microsc. Res. Tech. 70, 485-492 (2007).

19. T. Ng, A. Squire, G. Hansra, F. Bornancin, C. Prevostel, A. Hanby, W. Harris, D. Barnes, S. Schmidt, H. Mellor, P. I. Bastiaens, and P. J. Parker, Science 283, 2085-2089 (1999).

20. M. Peter and S. M. Ameer-Beg, Biol. Cell 96, 231-236 (2004).

21. J. Murphy, T. K. Sau, A. M. Gole, C. J. Orendorff, J. Gao, L. Gou, S. E. Hunyadi, and T. Li, J. Phys. Chem. B 109, 13857-13870 (2005).

22. F. Bestvater, E. Spiess, G. Stobrawa, M. Hacker, T. Feurer, T. Porwol, U. Berchner-Pfannschmidt, C. Wotzlaw, and H. Acker, J. Microsc. 208, 108-115 (2002).

23. A. Volkmer, D. A. Hatrick, and D. J. S. Birch, Meas. Sci. Technol. 8, 1339-49 (1997).

24. O. P. Varnavski, M. B. Mohamed, M. A. El-Sayed, and T. Goodson III, J. Phys. Chem. B 107, 3101-3104 (2003).

25. P. Biagioni, M. Celebrano, M. Savoini, G. Grancini, D. Brida, S. Mátéfi-Tempfli, M. Mátéfi-Tempfli, L. Duò, B. Hecht, G. Cerullo, and M. Finazzi, Phys. Rev. B 80, 045411 (2009). 